\title{
Canal y carne de corderos Santa Inés y cruces con Dorper o Texel: Un meta-análisis
}

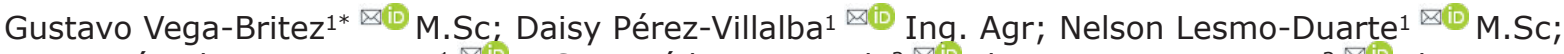 \\ José Velazquez-Duarte ${ }^{1}$ M.Sc; Ariádne Leonardo ${ }^{凶}$ M.D Ph.D; Marciana Retore ${ }^{凶}$ Ph.D; \\ Fernando de Vargas-Junior ${ }^{\otimes} \otimes$ Ph.D.
}

\begin{abstract}
${ }^{1}$ Universidad Nacional de Asunción, Facultad de Ciencias Agrarias, Orientación Producción Animal, Filial Pedro Juan Caballero. Pedro Juan Caballero, Paraguay.

2Universidade Federal da Grande Dourados, Faculdade de Ciências Agrárias, Departamento de Zootecnia, Dourados MS, Brasil. ${ }^{3}$ Embrapa Agropecuária Oeste, Manejo Animal, Dourados MS, Brasil.

*Correspondencia: gda_vega@hotmail.com
\end{abstract}

Recibido: Febrero 2020; Aceptado: Junio 2020; Publicado: Agosto 2020.

\section{RESUMEN}

Objetivo. Evaluar las características cuantitativas de la canal y composición química de la carne de corderos puros Santa Inés (SI) y cruces con Dorper (DO-SI) o Texel (TX-SI), terminados en confinamiento o semiconfinamiento, mediante un meta-análisis. Materiales y métodos. Bases de datos virtuales como Google Scholar, Science Direct y Scielo fueron utilizados para seleccionar los artículos científicos publicados desde el año 2000 a 2019. Palabras clave como genotipo, corderos, características de la canal y calidad de la carne, tanto en idioma español, portugués e inglés fueron utilizadas como criterios de inclusión de los artículos. De cada grupo genético, fueron evaluados: desempeño animal, características cuantitativas de la canal, cortes de la canal y composición química de la carne. Todos fueron incluidos en el análisis proveniente de 37 artículos científicos. Resultados. Peso corporal final, peso de la canal caliente, rendimiento de la canal fría y porcentaje de músculo no difieren entre los diferentes genotipos evaluados. Corderos DO-SI presentaron mayor consumo de materia seca, ganancia diaria de peso, área de ojo de lomo y porcentaje de lomo. Composición química no fue influenciada por los genotipos. Conclusiones. En ambiente tropical y subtropical, corderos puros SI terminados en confinamiento o semiconfinamiento presentan características cuantitativas de la canal y composición química similar a los corderos de cruces DO-SI y TX-SI.

Palabras clave: Calidad de la carne; confinamientos; ovinos; razas; rendimiento de la canal (Fuente: CAB).

\section{ABSTRACT}

Objective. To evaluate the quantitative characteristics of the carcass and chemical composition of the meat of purebred Santa Inês (SI) lambs and crosses with Dorper (DO-SI) or Texel (TX-SI), finished in feedlot or semi-feedlot, through a meta-analysis. Materials and methods. Virtual databases such as Google Scholar, Science Direct and Scielo were used to select scientific articles published from 2000 to 2019. Keywords such as genotype, lambs, carcass characteristics and meat quality, both in Spanish, Portuguese and English were used as criteria for the inclusion of articles. 
From each genetic group were evaluated: animal performance, quantitative characteristics of the carcass, carcass cuts and chemical composition of the meat. These variables were included in the analysis from 37 scientific articles. Results. Final body weight, hot carcass weight, cold carcass yield and muscle percentage do not differ between the different genetic groups evaluated. DO-SI lambs showed higher dry matter intake, daily weight gain, loin eye area and loin percentage. Chemical composition was not influenced by the genetic groups. Conclusions. In a tropical and subtropical environment, purebred lambs SI finished in feedlot or semi-feedlot have quantitative characteristics of the carcass and chemical composition similar to DO-SI and TX-SI lambs.

Keywords: Breeds; carcass yield; feedlot; meat quality; sheep (Source: $C A B$ ).

\section{INTRODUCCIÓN}

Se espera que, en un futuro próximo, debido al incremento de la población, además de aumentar la demanda de productos alimenticios, entre ella, la carne, los consumidores serán más exigentes en relación con la calidad del alimento, estos debiendo presentar adecuada información sobre su sistema de producción, alimentación, sustentabilidad y configurarse dentro de la norma de bienestar animal y medio ambiente (1).

Estos cuestionamientos, en ovinocultura, las investigaciones buscan responder sustentablemente, perfeccionando las prácticas o redescubriendo estrategias con el objetivo de ofrecer carne de calidad sostenible, ambientalmente segura y rentable al productor. La región central de América del Sur caracterizada por bioma Cerrado, parte del Pantanal y bosques atlánticos alberga una diversidad de genotipos de ovinos enfocado a la producción de carne, aunque con un nivel de especialización de los sistemas de producción todavía bajo (2).

Una de las estrategias en la búsqueda por ovinos de buena adaptación al clima y de alta producción es la combinación de genotipos mediante el cruzamiento. El uso de diferentes genotipos permite a los productores a optar de acuerdo a su objetivo de producción e infraestructura disponible, una vez que diferentes genotipos presentan variabilidad en la adaptación, conformación de la canal y madurez a diferentes edades de faena (3). El genotipo influencia en el aumento de peso y en las características cuantitativas de la canal, demostrando así que los cruces en los ovinos son necesarios para explorar la complementariedad de las razas y la heterosis es necesaria para lograr el mejor rendimiento y características deseables de la canal (4) y explorar positivamente los atributos de calidad de la carne (5). El cruzamiento puede constituir una herramienta útil en la producción de carne ovina, además de aumentar la rentabilidad de las granjas productoras (6).
Ovinos Santa Inés, constituye una de las razas localmente adaptada más importante en la producción ovina en el Brasil y se ha expandido al resto de Sudamérica, popularizado probablemente debido a su excepcional resistencia, fecundidad, habilidad materna y adaptabilidad a las diversas condiciones de suelo y clima. Esta raza es parte del patrimonio genético, histórico y cultural de Brasil, si son utilizados adecuadamente, de acuerdo con los diferentes entornos y sistemas de producción, pueden ser muy importantes en el desarrollo de la industria ovina (7).

Genotipos de ovinos como Texel y Dorper presentan estándares definidos y de gran perfil corporal para la producción de carne en el trópico $(8,9)$ aunque de estructura corporal más pequeña y compacta, mientras, la raza Santa Inés presenta bajo potencial para la producción de carne, sin embargo, su conformación corporal representa un animal rústico, que puede criarse en entornos con adversidades edafoclimáticas (9), por lo que su uso como matriz o raza materna con razas especializadas en carne como Dorper y Texel es ampliamente utilizado en el trópico.

Diferentes resultados son verificados en la literatura en relación a la influencia de los genotipos sobre las características de la canal y carne, la utilización del genotipo Dorper en cruzamientos con Santa Inés mejora la conformación (10) y la terminación de la canal y eleva el rendimiento de la misma (11) y produce carne con mayor acumulación de músculo y con menor cantidad de grasa en la canal, con un perfil nutricional más adecuado para el consumo humano (12), aunque, en confinamientos, el genotipo Santa Inés presenta similar desempeño y características de la canal en relación al cruce Santa Inés x Dorper (13).

Debido a la heterosis, probablemente, corderos de cruces Santa Inés con razas especializadas presenten mejores características de la canal 
y composición química de la carne diferentes. Sin embargo, constante mejoramiento genético contribuyó para la popularización de la raza Santa Inés, este hecho, algunas investigaciones presenta a esta raza en condiciones iguales en cuanto a características de la canal y composición química de la carne en relación con cruces con razas especializadas. Esto, levanta la hipótesis, si en la actualidad, es necesario el cruzamiento para la obtención de mejores resultados para las características de la canal y carne. Así mismo, aún faltan más estudios para la optimización de sistemas de producción existente y genotipos apropiados para la producción ovina en la región central de América del Sur (4). Por lo tanto, el objetivo del presente estudio fue evaluar las características cuantitativas de la canal y composición química de la carne de corderos puros Santa Inés y cruces con Dorper y Texel mediante un meta-análisis.

\section{MATERIALES Y MÉTODOS}

Revisión de literatura y criterios de inclusión. Fue realizada una investigación bibliográfica en bases de datos virtuales como Google Scholar, Science Direct y Scielo para seleccionar estudios que evalúan las características de la canal y composición química de la carne de corderos producidos en ambiente tropical y subtropical. Esta revisión fue realizada en cuatro etapas: identificación, selección, evaluación de elegibilidad e inclusión, siguiendo el flujo PRISMA (14) (Figura 1).

Palabras clave en español como; 1. "genotipo", 2. "corderos", 3. "calidad de la carne", 4. "características de la canal" en portugués como; 1. "genótipo", 2. "cordeiros", 3. "qualidade da carne", 4. "características da carcaça" y en inglés como: 1. "genotype", 2. "lambs", 3. "meat quality", 4. "carcass characteristic", fueron utilizadas como criterios de inclusión para la búsquedas bibliográficas, además de ser conducidos en ambiente tropical y subtropical. Artículos publicados entre los años 2000 a 2019 fueron seleccionados para conformar los datos iniciales de la investigación.

Después de la búsqueda, fueron encontrados y seleccionados 90 artículos de interés por contener por lo menos una de las palabras claves en el título o resumen. Seguidamente, en la selección y evaluación, fueron leídos los resúmenes y excluidos 28 artículos por tratarse de investigaciones realizadas en ambiente templado o árido.

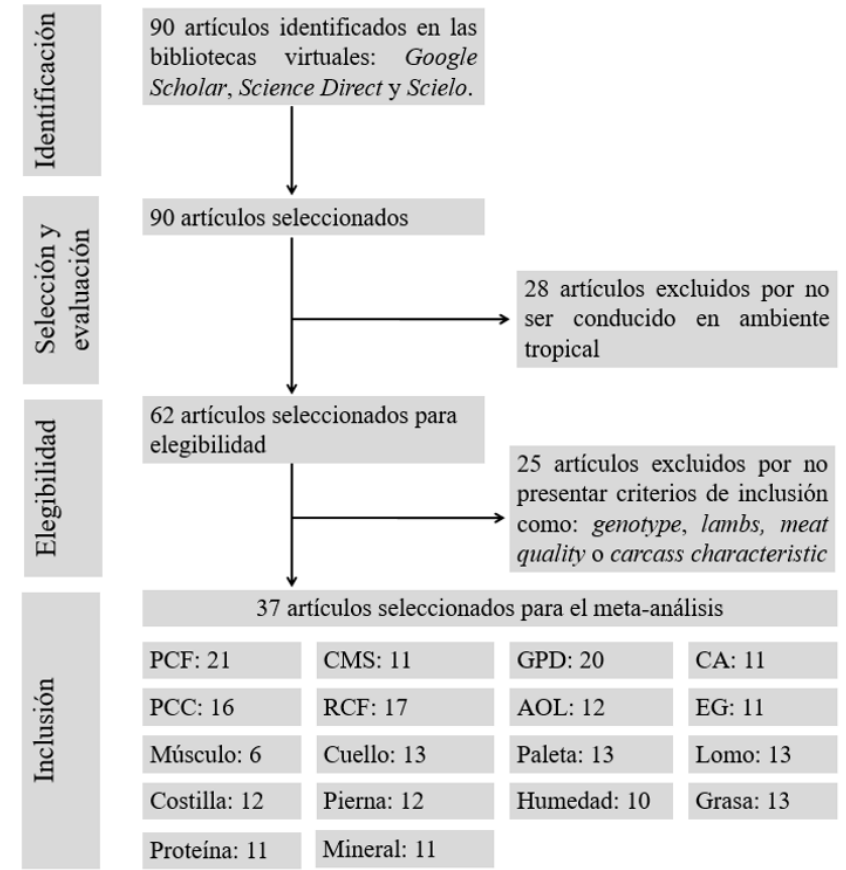

Figura 1. Diagrama de flujo PRISMA (Preferred Reporting Items for Systematic Reviews and Meta-Analyses). PCF: peso corporal final; CMS: consumo de materia seca; GPD: ganancia promedio diario de peso; CA: conversión alimentar; PCC: peso de la canal caliente; RCF: rendimiento de la canal fría; AOL: área de ojo de lomo; EG: espesura de la grasa, porcentajes de músculo, cuello, paleta, lomo, costilla y pierna; y composición química de la carne (gr/ 100gr): humedad, grasas, proteína y mineral.

Así, 62 artículos conformaron la elegibilidad, que fueron leídas integralmente, de acuerdo a los criterios de elegibilidad predefinidos. Después de esa etapa, 25 artículos fueron excluidos por no presentar las palabras clave "genotype", "lambs", "meat quality" o "carcass characteristic" como variables estudiadas, así, 37 estudios fueron seleccionados para el metaanálisis (Figura 1).

Tabulación de los datos. Los datos de los diferentes artículos fueron clasificados de acuerdo con los genotipos y variables de interés. En total, tres diferentes genotipos fueron evaluados y son: Santa Inés (SI), Dorper x Santa Inés (DO-SI) y Texel x Santa Inés (TX-SI). De cada genotipo, el desempeño animal, las características cuantitativas de la canal, cortes de la canal y composición química de la carne fueron incluidos en el análisis (Tabla 1). Las informaciones extraídas de los artículos, de la parte de resumen, materiales y métodos y resultados y discusión fueron organizadas en una planilla. 
Vega-Britez et al - Canal y carne de corderos Santa Inés y cruces con Dorper o Texel

Tabla 1. Descripción del estudio primario incluido en el meta-análisis $(n=37)$.

\begin{tabular}{|c|c|c|c|}
\hline Estudios & País & Sistema de alimentación & Variables \\
\hline$(4)$ & Brasil & Confinamiento & PCF, CMS, GPD, CA, PCC, RCF, AOL \\
\hline$(5)$ & Brasil & Confinamiento & PCC, Humedad, Grasas, Proteína, Mineral \\
\hline$(10)$ & Brasil & Confinamiento & PCF, PCC, AOL, EG \\
\hline$(11)$ & Brasil & Confinamiento & GPD, PCC, RCF, AOL, EG, Cuello, Paleta, Lomo, Costilla, Pierna \\
\hline$(12)$ & Brasil & Confinamiento & $\begin{array}{c}\text { PCF, CMS, GPD, CA, PCC, RCF, AOL, Músculo, Cuello, Paleta, Lomo, } \\
\text { Costilla, Pierna, Grasas, Proteína, Mineral }\end{array}$ \\
\hline$(13)$ & Brasil & Confinamiento & PCF, GPD, PCC, RCF, EG, Cuello, Paleta, Lomo, Costilla, Pierna \\
\hline$(15)$ & Brasil & Confinamiento & PCF, PCC, RCF, AOL, EG, Cuello, Paleta, Lomo, Costilla, Pierna \\
\hline$(16)$ & Brasil & Confinamiento & PCF, CMS, GPD, RCF, Cuello, Paleta, Lomo, Costilla, Pierna \\
\hline$(17)$ & Brasil & Confinamiento & PCF, CMS, GPD, CA, PCC, RCF, AOL, EG, Cuello, Paleta, Lomo, Pierna \\
\hline$(18)$ & Brasil & Confinamiento & CMS, GPD \\
\hline$(19)$ & Brasil & Confinamiento & PCF, GPD \\
\hline$(20)$ & Brasil & Semi-confinamiento & PCF, GPD, PCC, RCF, EG, Cuello, Paleta, Lomo, Costilla, Pierna \\
\hline$(21)$ & Brasil & Confinamiento & PCF, CMS, GPD, CA, RCF \\
\hline$(22)$ & Brasil & Confinamiento & $\begin{array}{c}\text { PCF, CMS, GPD, CA, PCC, RCF, AOL, EG, Cuello, Paleta, Lomo, } \\
\text { Costilla, Pierna }\end{array}$ \\
\hline$(23)$ & Brasil & Semi-confinamiento & AOL, EG, Músculo \\
\hline (24) & Brasil & Confinamiento & PCF, GPD, Humedad, Grasas, Proteína, Mineral \\
\hline$(25)$ & Brasil & Confinamiento & PCF, CMS, GPD, CA \\
\hline$(26)$ & Brasil & Confinamiento & PCF, PCC, AOL, EG, Músculo, Cuello, Paleta, Lomo, Costilla, Pierna \\
\hline$(27)$ & Brasil & Confinamiento & PCF, AOL, Músculo \\
\hline$(28)$ & Brasil & Confinamiento & Humedad, Proteína, Grasas, Mineral \\
\hline (29) & Brasil & Confinamiento & Grasas \\
\hline$(30)$ & Brasil & Confinamiento & Humedad, Grasas, Proteína, Mineral \\
\hline$(31)$ & Brasil & Confinamiento & Humedad, Grasas, Proteína, Mineral \\
\hline$(32)$ & Brasil & Confinamiento & Humedad, Grasas, Proteína, Mineral \\
\hline$(33)$ & Brasil & Confinamiento & PCF, CMS, GPD, CA \\
\hline (34) & Brasil & Semi-confinamiento & AOL, Músculo, Grasas, Proteína, Mineral \\
\hline$(35)$ & Brasil & - & Humedad, Grasas, Proteína, Mineral \\
\hline$(36)$ & Brasil & Confinamiento & PCF, RCF, PCC, EG \\
\hline$(37)$ & Brasil & Confinamiento & PCF, GPD \\
\hline$(38)$ & Brasil & Confinamiento & Músculo, Cuello, Paleta, Lomo, Costilla, Pierna \\
\hline (39) & Brasil & Confinamiento & Cuello, Paleta, Lomo, Costilla, Pierna \\
\hline$(40)$ & Brasil & Confinamiento & PCC, RCF, Cuello, Paleta, Lomo, Costilla, Pierna \\
\hline$(41)$ & Brasil & Semi-confinamiento & PCF, PCC, RCF, Cuello, Paleta, Lomo, Costilla, Pierna \\
\hline$(42)$ & Brasil & Semi-confinamiento & Humedad, Grasas, Proteína, Mineral \\
\hline$(43)$ & Brasil & Semi-confinamiento & PCF, PCC, RCF \\
\hline$(44)$ & Brasil & Semi-confinamiento & $\mathrm{PCF}, \mathrm{CMS}, \mathrm{GPD}, \mathrm{CA}, \mathrm{PCC}, \mathrm{RCF}$ \\
\hline$(45)$ & Brasil & Semi-confinamiento & PCF, CMS, GPD, PCC, RCF, AOL, EG, Cuello, Paleta, Lomo, Costilla, Pierna \\
\hline
\end{tabular}

PCF: peso corporal final; CMS: consumo de materia seca; GPD: ganancia promedio de peso diario; PCC: peso de la canal caliente; RCF: rendimiento de la canal fría; AOL: área de ojo de lomo; EG: espesura de la grasa de la canal; CA: conversión alimentar.

En las características de la canal fueron seleccionados el peso corporal final (PCF), consumo de materia seca (CMS), ganancia promedio diario de peso (GPD), conversión alimentar (CA), peso de la canal caliente (PCC), rendimiento de la canal fría (RCF), área de ojo de lomo (AOL), espesura de la grasa (EG), y los porcentajes de músculo, cuello, paleta, lomo, costilla y pierna. Composición química de la carne como humedad, grasas, proteína y mineral también fueron evaluados en los distintos genotipos. 
Vega-Britez et al - Canal y carne de corderos Santa Inés y cruces con Dorper o Texel

Análisis estadísticos. Los análisis fueron realizados de acuerdo con el siguiente modelo matemático general:

$Y_{i j k}=\mu+T_{i}+a_{j}+\varepsilon_{i j k \prime}$

en que $\gamma_{i j k}=$ variables dependientes, $\mu=$ promedio de todas las observaciones, $T_{i}=$ efecto fijo de los genotipos, $a_{j}=$ efecto aleatorio de los artículos $y$ $\varepsilon_{\mathrm{ijk}}=$ error residual aleatorio.

Después del test de normalidad de los residuos, un análisis de variancia fue realizada utilizando el programa estadístico AgroEstat ${ }^{\circledR}$. Test de comparación de promedio de Tukey al 5\% de probabilidad fue utilizado. Variables como CMS, PCF, RCF, GPD, paleta, pierna y mineral fueron trasformados a través de log 10 , porque no presentaron normalidad.

\section{RESULTADOS}

La distribución de la cantidad de corderos según las diferentes variables se detalla en la Tabla 2 . En las investigaciones publicadas, corderos de cruces DO-SI es más frecuente en relación con cruces TX-SI, es decir, este último con menor cantidad de corderos evaluados para cada una de las variables en el presente meta-análisis. Promedios de días en confinamiento o semiconfinamiento entre los genotipos SI, DO-SI y TX-SI fueron similares.

Los distintos genotipos evaluados presentaron mismo peso de faena (PCF, $p>0.05$ ) variando de $32.02 \mathrm{~kg}$ para SI, $33.03 \mathrm{~kg}$ para el cruce TX-SI y 33.41 para DO-SI (Tabla 3). El consumo de materia seca ( $\mathrm{gr} / \mathrm{d})$ es significativamente influenciado por los genotipos $(p \leq 0.05)$, siendo superior en los cruces DO-SI con $1.144,3 \mathrm{gr}$, mientras, SI presenta consumo diario de $1.003,3 \mathrm{gr}$ y TX-SI el menor consumo con $842.5 \mathrm{gr}$ (Tabla 3 ).

Ganancia promedio de peso diario (GPD) es superior $(p \leq 0.05)$ en corderos de cruces DO-SI con $0.230 \mathrm{~kg} / \mathrm{d}$, mientras corderos SI presentan GPD de $0.194 \mathrm{~kg} / \mathrm{d}$ y TX-SI de $0.160 \mathrm{~kg} / \mathrm{d}$. Conversión alimentar (CA), peso de la canal caliente (PCC), rendimiento de la canal fría (RCF) y porcentaje de músculo no fueron influenciados $(p>0.05)$ por los genotipos evaluados, mientras, el área de ojo de lomo (AOL) y espesura de la grasa de la canal (EG) fueron influenciados, siendo mayor en los cruces DO-SI con 13.69 $\mathrm{cm}^{2}$ y 2,97 mm, mientras menor para SI, con $11.62 \mathrm{~cm}^{2}$ de $A O L$, siendo la EG menor en SI y TX-SI (Tabla 3).
Tabla 2. Número de repeticiones de corderos para las diferentes variables según los genotipos evaluados.

\begin{tabular}{lccccc}
\hline \multirow{2}{*}{ Variables } & \multicolumn{4}{c}{ Genotipos } & \multirow{5}{*}{ Total } \\
\cline { 2 - 6 } & SI & DO-SITX-SI & \\
\hline Pesempeño animal & \\
Peso corporal final (PCF, kg) & 414 & 329 & 141 & 884 \\
Consumo de materia seca (CMS, gr/día) & 114 & 136 & 68 & 318 \\
Ganancia de peso diario (GPD, gr/día) & 304 & 231 & 170 & 705 \\
Conversión alimentar (CA, kg/kg) & 136 & 100 & 96 & 332
\end{tabular}

Cuantitativa de la canal

Peso de la canal caliente (PCC, kg) $286 \quad 201 \quad 135 \quad 622$

Rendimiento de la canal fría (RCF, \%) $300 \quad 199 \quad 151 \quad 650$

Área de ojo de lomo $\left(\mathrm{AOL}, \mathrm{cm}^{2}\right) \quad 199 \quad 149 \quad 75 \quad 423$

Músculo, \% $109 \quad 61 \quad 45 \quad 215$

Espesura de la grasa (EG, mm) $\quad 194 \quad 178 \quad 94 \quad 466$

Cortes de la canal

$\begin{array}{lcccc}\text { Cuello, \% } & 189 & 137 & 83 & 409 \\ \text { Paleta, \% } & 189 & 149 & 83 & 421 \\ \text { Lomo, \% } & 201 & 149 & 83 & 433 \\ \text { Costilla, \% } & 191 & 129 & 83 & 403 \\ \text { Pierna, \% } & 201 & 149 & 105 & 455\end{array}$

Composición química de la carne

Humedad (gr/100 gr) $\quad 152 \quad 96 \quad 54 \quad 302$

Grasas (gr/100 gr) $\quad 215 \quad 157 \quad 71 \quad 443$

Proteína (gr/100 gr) $\quad \begin{array}{lllll}160 & 122 & 54 & 336\end{array}$

Mineral (gr/100 gr) $\quad \begin{array}{llll}160 & 122 & 54 & 336\end{array}$

Días en confinamiento o semi-confinamiento

$\begin{array}{cccc}\text { Mínimo } & 36.75 & 35.87 & 45 \\ \text { Promedio } & 89.31 & 79.32 & 95.42\end{array}$

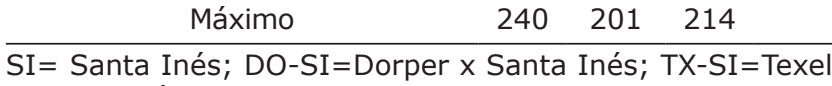
$x$ Santa Inés.

Los resultados obtenidos demuestran la importancia del cruzamiento en la obtención de mejores GPD y AOL. Sin embargo, para las demás variables cuantitativas de la canal evaluadas, el genotipo no ejerce influencia significativa, indicando que la elección de uno u otro agrupamiento genético puede ser acorde al gusto del productor. Aunque la GPD fue mayor para DO-SI en similares días de confinamiento o semiconfinamiento (Tabla 2 ), esto no refleja en mayor porcentaje de músculo ( $p>0.05)$, por ende, en valores absoluto, fue mayor para cruces DO-SI, es probable que la diferencia de peso sea expresada en el aumento de la espesura de la grasa de la canal $(2,97 \mathrm{~mm})$ de los animales DOSI en relación a SI (1.94 mm) y TX-SI $(1.79 \mathrm{~mm})$. 
Vega-Britez et al - Canal y carne de corderos Santa Inés y cruces con Dorper o Texel

Tabla 3. Desempeño animal y características cuantitativas de la canal de diferentes genotipos de corderos.

\begin{tabular}{ccccccc}
\hline Variable & SI & $\begin{array}{c}\text { Genotipos } \\
\text { DO-SI }\end{array}$ & TX-SI & EPM & Valor - p & $\mathbf{n}^{\mathbf{1}}$ \\
\hline PCF $(\mathrm{kg})$ & 32.02 & 33.41 & 33.03 & 0.927 & 0.640 & 884 \\
CMS $(\mathrm{gr} / \mathrm{d})$ & $1003.3 \mathrm{a}$ & $1144.3 \mathrm{a}$ & $842.5 \mathrm{~b}$ & 0.017 & 0.001 & 318 \\
GPD $(\mathrm{kg} / \mathrm{d})$ & $0.194 \mathrm{ab}$ & $0.230 \mathrm{a}$ & $0.160 \mathrm{~b}$ & 0.011 & 0.036 & 705 \\
CA $(\mathrm{kg} / \mathrm{kg})$ & 6.06 & 4.51 & 6.04 & 0.455 & 0.065 & 332 \\
PCC $(\mathrm{kg})$ & 15.23 & 15.83 & 15.06 & 0.683 & 0.798 & 622 \\
RCF $(\%)$ & 46.83 & 47.56 & 45.34 & 0.685 & 0.208 & 650 \\
AOL $\left(\mathrm{cm}^{2}\right)$ & $11.62 \mathrm{~b}$ & $13.69 \mathrm{a}$ & $13.18 \mathrm{a}$ & 0.413 & 0.019 & 423 \\
EG $(\mathrm{mm})$ & $1.94 \mathrm{~b}$ & $2.97 \mathrm{a}$ & $1.79 \mathrm{~b}$ & 0.270 & 0.009 & 466 \\
Músculo $(\%)$ & 55.12 & 59.47 & 51.04 & 3.478 & 0.585 & 215 \\
\hline
\end{tabular}

SI= Santa Inés; DO-SI=Dorper $\times$ Santa Inés; TX-SI=Texel x Santa Inés.

${ }^{1} \mathrm{n}=$ número de repeticiones, total de corderos.

a,b Los valores dentro de una fila con letras diferentes difieren significativamente a 5\% de probabilidad según Test de Tukey. $\mathrm{EPM}=$ error padrón de la media; $\mathrm{PCF}=$ peso corporal final; $\mathrm{CMS}=$ consumo de materia de seca; $\mathrm{GPD}=\mathrm{ganancia}$ promedio diario de peso; $C A=$ conversión alimentar; $P C C=$ peso de canal caliente; RCF; rendimiento de canal fría; $A O L=$ área de ojo del lomo; $\mathrm{EG}=$ espesura de la grasa de la canal.

El lomo (\%) fue el único corte del canal influenciado por los genotipos $(p \leq 0.05)$, siendo mayor en el cruce DO-SI, con $11.84 \%$, seguido por SI con $8.82 \%$ y TX-SI con $6.39 \%$, mientras, el cuello, paleta, costilla y la pierna no fueron influenciados $(p>0.05)$ por los genotipos evaluados (Tabla 4).

El genotipo no ejerce influencia sobre el corte de la canal de mayor interés como pierna. Estos indican que todos los agrupamientos genéticos evaluados pueden presentar resultados similares para corte de la canal.

La composición química de la carne de corderos no fue influenciada $(p>0.05)$ por los genotipos evaluados (Tabla 5). Esto indica que las variables evaluadas son independientes del genotipo.

Tabla 4. Cortes de la canal de diferentes genotipos de corderos.

\begin{tabular}{ccccccc}
\hline Variable & SI & $\begin{array}{c}\text { Genotipos } \\
\text { DO-SI }\end{array}$ & TX-SI & EPM & Valor - $\mathbf{p}$ & $\mathbf{n}^{\mathbf{1}}$ \\
\hline Cuello (\%) & 8.25 & 7.06 & 7.33 & 0.348 & 0.131 & 409 \\
Paleta (\%) & 16.93 & 17.76 & 15.36 & 0.955 & 0.232 & 421 \\
Lomo (\%) & $8.82 \mathrm{~b}$ & $11.84 \mathrm{a}$ & $6.39 \mathrm{~b}$ & 0.758 & 0.005 & 433 \\
Costilla (\%) & 27.67 & 29.31 & 25.97 & 1478 & 0.565 & 403 \\
Pierna (\%) & 29.06 & 32.16 & 27.55 & 1634 & 0.161 & 455 \\
\hline
\end{tabular}

SI = Santa Inés; DO-SI=Dorper $\times$ Santa Inés; TX-SI=Texel x Santa Inés.

$1 \mathrm{n}=$ número de repeticiones, total de corderos.

a,b Los valores dentro de una fila con letras diferentes difieren significativamente a 5\% de probabilidad según Test de Tukey. $E P M=$ error padrón de la media.

Tabla 5. Composición química (gr/100 gr) del músculo longissimus dorsi de diferentes genotipos de corderos.

\begin{tabular}{|c|c|c|c|c|c|c|}
\hline \multirow{2}{*}{ Variables } & \multicolumn{3}{|c|}{ Genotipos } & \multirow{2}{*}{ EPM } & \multirow{2}{*}{ Valor - p } & \multirow{2}{*}{$n^{1}$} \\
\hline & SI & DO-SI & TX-SI & & & \\
\hline Humedad & 74.05 & 73.61 & 75.29 & 0.451 & 0.387 & 302 \\
\hline Grasas & 3.07 & 3.56 & 3.49 & 0.335 & 0.733 & 443 \\
\hline Proteína & 21.63 & 21.54 & 20.40 & 0.528 & 0.642 & 336 \\
\hline Mineral & 1.13 & 1.15 & 1.30 & 0.052 & 0.425 & 336 \\
\hline
\end{tabular}

$\mathrm{SI}=$ Santa Inés; DO-SI=Dorper $\times$ Santa Inés; TX-SI=Texel $\times$ Santa Inés. ${ }^{1} \mathrm{n}=$ número de repeticiones, total de corderos. $\mathrm{EPM}=$ error padrón de la media. 
Vega-Britez et al - Canal y carne de corderos Santa Inés y cruces con Dorper o Texel

\section{DISCUSIÓN}

La sumarización de los datos permite caracterizar mejor a los genotipos de mayor desempeño en el CMS, GPD, AOL y EG, siendo estos superiores en cruces DO-SI (Tabla 3), aunque en otras investigaciones $(10,15,16,17,18,19,20,21)$ no se constataron diferencias significativas para GPD y RCF entre genotipos DO-SI y SI, sin embargo, son relatados mayor CMS en corderos DO-SI en relación a SI $(16,18,22)$, no obstante otros no verificaron diferencias al evaluar los mismos genotipos (21). Por otro lado, no observaron diferencias para las variables PCF, CMS, GPD, CA, PCC, RCF entre genotipos DO-SI y SI, pero se verificaron mayor AOL en cruces DO-SI (12), mientras, son observadas diferencias para AOL entre corderos SI y TX-SI (23).

Diferencias significativas no fueron verificadas para GPD entre cruces DO-SI y SI $(17,18,19,24,25)$, aunque otros constataron diferencias entre cruces DO-SI y SI (22). Así mismo, diferencias significativas para GPD al evaluar entre genotipos DO-SI, SI y TX-SI no fueron verificadas (20). Mayor GPD observada para DO-SI y SI en relación a TX-SI (Tabla 3 ) no refleja en mayor porcentaje de músculo, esta diferencia de ganancia de peso puede ser expresada en mayor EG de la canal. En otra investigación, no fueron observadas diferencias significativas para PCF y AOL para corderos SI y DO-SI, sin embargo, cruces DO-SI presentaron mayor EG (10), así mismo, el AOL y EG no presentan correlación significativa (46). Esto, indica que corderos SI presentan canal más magra sin afectar el RCF y el acabamiento de la canal.

Aunque los genotipos evaluados presentaron tiempos promedios en días en confinamiento o semiconfinamiento similares (Tabla 2), los mismos presentan momentos fisiológicos diferentes (mayor EG en cruces DO-SI), en ese sentido, el criterio para la faena debe ser en base al acabamiento de la canal y no en tiempo fijo de terminación (47).

Corderos puros SI presentan rendimiento y características de canal similares a los corderos DO-SI y TX-SI (13). Sin embargo, corderos TX-SI presentan mejor potencial para características de calidad de la canal y de la carne en relación con corderos SI (23). AOL superiores son verificados en corderos oriundos de cruces TX-SI en relación con los corderos SI (11). El peso ideal de faena de los corderos es a los $35 \mathrm{~kg}$ peso vivo, momento en que se observan mayores rendimientos de la carne en las mismas y mayor EG comparado a corderos faenados a los $30 \mathrm{~kg}$ (11). Este dato se refleja en el resultado obtenido (Tabla 3), al verificar que el PCF varía de $32.02 \mathrm{~kg}$ para SI, $33.03 \mathrm{~kg}$ para TX-SI y 33.41 para DO-SI, es decir, fueron faenados en el momento de mayor rendimiento de la canal.

Variaciones no fueron observadas para AOL en corderos de cruces DO-SI y SI en tres condiciones corporales evaluadas, magro, intermedio y gordo (15), ni en función a dos niveles de energía en la dieta $(10,26)$, aunque en otras investigaciones, fueron observados diferencias significativas para AOL, siendo superior en los corderos de cruces DO-SI en relación a genotipo SI $(17,22,27)$.

Resultados similares (Tabla 4) fueron observados en corderos DO-SI y SI, excepto para lomo, siendo este corte proporcionalmente superior en cruces DO-SI (16). La homogeneidad en relación con las proporciones de los cortes tal vez se debe a las características morfométricas similares relatadas entre corderos de cruces DO-SI y SI (48), aunque difieren de los obtenidos en otra investigación, donde fueron verificados mayor porcentaje de paleta en corderos SI en relación a cruces DOSI (22). Mayor porcentaje de paleta sugiere que el animal tuvo mayor desplazamiento en busca de alimentos para satisfacer su requerimiento nutricional diario (49).

Aunque diversos estudios $(11,17,26)$ verificaron que el cruzamiento permite mejor desempeño que las razas puras, principalmente en cuanto a rendimientos de los cortes de la canal, sin embargo, en este meta-análisis, la sumarización permite equiparar mejor los datos, demostrando que para la proporción de los cortes de la canal, excepto para lomo, el genotipo puro SI presenta resultados semejantes a los cruces DO-SI y TX-SI, estos sugiere el avance del mejoramiento genético en animales de pelo o deslanado para la especialización en producción de carne, principalmente cuando terminado en confinamiento, considerando que la mayoría de los estudios analizados provienen del mencionado sistema (Tabla 1).

Mayor CMS y GPD verificado en los corderos DOSI (Tabla 3) no se refleja estadísticamente en mejor conversión alimentar en el mismo genotipo, aunque, numéricamente fue la mejor conversión (4.51 para DO-SI vs. 6.06 para SI y 6.04 para TX-SI). Así mismo, el AOL mayor verificado para DO-SI $\left(13.69 \mathrm{~cm}^{2}\right)$, estadísticamente no condice con una mayor acumulación de músculo 
(59.47\% ver Tabla 3) o pierna (32.16\%, Tabla 4), aunque numéricamente se observa diferencias, sin embargo, el mayor AOL observado para corderos DO-SI parece estar relacionado a un mayor porcentaje de lomo (11.84\%, Tabla 4).

Esta inconsistencia para algunas de las variables tal vez se debe a la cantidad de datos obtenidos y analizados para cada uno de ellos, considerando que fue utilizado 11 artículos para CMS, 20 para GPD, 12 para AOL, 6 para músculo y 12 para pierna (Figura 1, Tablas 1 y 2 ).

No fueron observados diferencias estadísticas para composición química de la carne de corderos entre genotipos DO-SI y SI $(18,23,30,31)$. En ese sentido, ni raza, el sexo (32) o niveles de proteína bruta en la dieta (33) ejercen influencia sobre la composición química de la carne de corderos SI y cruces DO-SI, aunque, en otra investigación, mayor concentración de grasas en cruces DO-SI que corderos SI fueron verificados (34).

Inicialmente el enfoque del meta-análisis era más amplio, tal es así, que en las palabras clave de búsqueda no incluyó "Santa Inés". Esto es indicativo del nivel de importancia en los últimos 20 años de la raza Santa Inés y el número limitado en estas últimas dos décadas de investigaciones publicadas de forma significativa de otras razas. En ese sentido, es importante destacar que el presente meta-análisis no limitó las búsquedas a investigaciones realizadas en Brasil, no obstante, se puede observar en la Tabla 1 que, al azar, todos los artículos que reunieron los criterios de inclusión fueron desarrollados allí, a pesar de que esta raza también es criada en otros países.
En conclusión, corderos Santa Inés terminados en confinamiento o semiconfinamiento presentan similares características cuantitativas de la canal y composición química de la carne en relación con corderos del cruce Dorper x Santa Inés y Texel x Santa Inés.

Corderos Dorper x Santa Inés se destacan por mayor consumo de materia seca, ganancia de peso diario, área de ojo de lomo, espesura de la grasa y porcentaje de lomo.

Corderos Santa Inés presenta promedios de días en confinamiento o semiconfinamiento similar al cruce Dorper x Santa Inés.

Nivel de acabamiento de la canal debe ser el criterio adoptado para la faena de los genotipos Santa Inés, Dorper x Santa Inés y Texel x Santa Inés, no así el tiempo fijo de terminación.

Corderos Santa Inés esta entre la más utilizada en experimentos que evalúan desempeño, rendimiento de la canal y calidad de la carne en Brasil, presentando canal más magra sin perjudicar el rendimiento y acabamiento de la misma.

\section{Conflicto de intereses}

Los autores del presente estudio declaramos que no existe conflicto de intereses con la publicación de este manuscrito.

\section{REFERENCIAS}

1. Ponnampalam EN, Bekhit AED, Bruce $H$, Scollan ND, Muchenje V, Silva $P$, et al. Production strategies and processing systems of meat: Current status and future outlook for innovation - A global perspective. In C. Galanakis (Ed.). Sustainable meat production and processing. Cambridge, Massachusetts: Academic Press; 2019; https://doi.org/10.1016/B978-0-12814874-7.00002-X
2. Raineri C, Nunes BCP, Gameiro AH. Technological characterization of sheep production systems in Brazil. Anim Sci J. 2015; 86(4):476-485. https://doi. org/10.1111/asj.12313

3. Figueiredo GC, Rezende MPG, Figueiredo MP, Bozzi R, Souza Junior AAO, Carneiro PLS, et al. Morphofunctional characteristics of Dorper sheep crossed with Brazilian native breeds. Small Rumin Res. 2019; 170:143-148. https://doi.org/10.1016/j. smallrumres.2018.11.024 
Vega-Britez et al - Canal y carne de corderos Santa Inés y cruces con Dorper o Texel

4. Nunes IA, Brito RAM, Morais LA de, Silva VS, Silva MAP de, Carmo RM. Performance, carcass characteristics, and centesimal composition of meat from Santa Inês lambs and Texel crossbred lambs (Santa Inês $\times$ Texel). Can J Anim Sci. 2019; 99(2):228-236. https://doi.org/10.1139/ cjas-2016-0231

5. Monaco CA, Freire MTA, Melo L, Rosa AF, Carrer CC, Trindade MA. Eating quality of meat from six lamb breed types raised in Brazil. J Sci Food Agric. 2015; 95(8):17471752. https://doi.org/10.1002/jsfa.6894

6. Blasco M, Campo MM, Balado J, Sañudo C. Effect of Texel crossbreeding on productive traits, carcass and meat quality of Segureña lambs. J Sci Food Agric. 2019; 99(7):33353342. https://doi.org/10.1002/jsfa.9549

7. Ribeiro ELA, González-García E. Indigenous sheep breeds in Brazil: potential role for contributing to the sustainability of production systems. Trop Anim Health Prod. 2016; 48(7):1305-1313. https://doi. org/10.1007/s11250-016-1109-3

8. Kiya CK, Pedrosa VB, Muniz KFA, Gusmão $A L$, Pinto LFB. Estimates of the genetic parameters of a Dorper flock in Brazil. Small Rumin Res. 2019; 171:57-62. https://doi. org/10.1016/j.smallrumres.2018.12.007

9. Ramos IO, Rezende MPG, Carneiro PLS, Souza JC, Sereno JR, Bozzi R, et al. Body conformation of Santa Inês, Texel and Suffolk ewes raised in the Brazilian Pantanal. Small Rumin Res. 2019; 172:42-47. https://doi. org/10.1016/j.smallrumres.2019.01.011

10. Cartaxo FQ, Sousa WH, Cezar MF, Costa RG, Cunha MGG, Gonzaga Neto S. Características de carcaça determinadas por ultrassonografia em tempo real e pós-abate de cordeiros terminados em confinamento com diferentes níveis de energía na dieta. R Bras Zootec. 2011; 40(1):160-167. http://dx.doi.org/10.1590/ $\underline{\text { s1516-35982011000100023 }}$

11. Cardoso MTM, Landim AV, Louvandini H, McManus C. Performance and carcass quality in three genetic groups of sheep in Brazil. R Bras Zootec. 2013; 42(10):734742. http://dx.doi.org/10.1590/S1516$\underline{35982013001000007}$
12. Issakowicz J, Issakowicz ACKS, Bueno MS, Costa RLD, Geraldo AT, Abdalla AL, et al. Crossbreeding locally adapted hair sheep to improve productivity and meat quality. Sci Agric. 2018; 75(4):288-295. http://dx.doi. org/10.1590/1678-992x-2016-0505

13. Garcia IFF, Costa TIR, Almeida AK, Pereira IG, Alvarenga FAP, Lima NLL. Performance and carcass characteristics of Santa Inês pure lambs and crosses with Dorper e Texel at different management systems. R Bras Zootec. 2010; 39(6):1313-1321. http://dx.doi.org/10.1590/S1516$\underline{35982010000600021}$

14. Moher D, Liberati A, Tetzlaff J, Altman DG, Prisma Group. Preferred Reporting Items for Systematic Reviews and Meta-Analyses: The PRISMA Statement. Ann Intern Med. 2009; 151(4):264-269. https://annals.org/aim/ article-abstract/744664

15. Cartaxo FQ, Cezar MF, Sousa WH, Gonzaga Neto S, Pereira Filho JM, Cunha MGG. Características quantitativas da carcaça de cordeiros terminados em confinamento e abatidos em diferentes condições corporais. R Bras Zootec. 2009; 38(4):697704. http://dx.doi.org/10.1590/S1516$\underline{35982009000400016}$

16. Araújo Filho JT, Costa RG, Fraga $A B$, Sousa WH, Cezar MF, Batista ASM. Desempenho e composição da carcaça de cordeiros deslanados terminados em confinamento com diferentes dietas. R Bras Zootec. 2010; 39(2):363-371. http://dx.doi.org/10.1590/ S1516-35982010000200020

17. Cartaxo FQ, Sousa WH, Cezar MF, Cunha MGG, Menezes LL, Ramos JPF, et al. Desempenho e características de carcaça de cordeiros Santa Inês e suas cruzas com Dorper terminados em confinamento. Rev Bras Saúde Prod Anim. 2017; 18(2):388401. http://dx.doi.org/10.1590/s1519$\underline{99402017000200017}$

18. Costa RG, Batista ASM, Madruga MS, Gonzaga Neto S, Queiroga RCRE, Araújo Filho JT, et al. Physical and chemical characterization of lamb meat from different genotypes submitted to diet with different fibre contents. Small Rumin Res. 2009; 81:29-34. https://doi.org/10.1016/j. smallrumres.2008.10.007 
19. Costa RG, Santos NM, Sousa WH, Queiroga RCRE, Azevedo PS, Cartaxo FQ. Qualidade física e sensorial da carne de cordeiros de três genótipos alimentados com rações formuladas com duas relações volumoso: concentrado. R Bras Zootec. 2011; 40(8):1781-1787. http://dx.doi. org/10.1590/S1516-35982011000800023

20. Prado Paim T, Silva AF, Martins RFS, Borges BO, Lima PMT, Cardoso CC, et al. Performance, survivability and carcass traits of crossbred lambs from five paternal breeds with local hair breed Santa Inês ewes. Small Rumin Res. 2013; 112:28-34. https://doi. org/10.1016/j.smallrumres.2012.12.024

21. Sousa WH, Cartaxo FQ, Costa RG, Cezar MF, Cunha MGG, Pereira Filho JM, et al. Biological and economic performance of feedlot lambs feeding on diets with different energy densities. R Bras Zootec. 2012; 41(5):1285-1291. http://dx.doi. org/10.1590/S1516-35982012000500028

22. Souza DA, Selaive-Villarroel AB, Pereira ES, Silva EMC, Oliveira RL. Effect of the Dorper breed on the performance, carcass and meat traits of lambs bred from Santa Inês sheep. Small Rumin Res. 2016; 145:76-80. https://doi.org/10.1016/j. smallrumres.2016.10.017

23. Landim AV, Vasconcelos AM, Corrêa MP, Costa HHA, Fioravanti MCS, Louvandini H, et al. Tissue and centesimal composition of the $12^{\text {th }}$ rib of lambs from genetic groups different. Rev Bras Saúde Prod Anim. 2015; 16(2):470-479. http://dx.doi.org/10.1590/ $\underline{\mathrm{S} 1519-99402015000200004}$

24. Costa RG, Santos NM, Queiroga RCRE, Sousa WS, Madruga MS, Cartaxo FQ. Physicochemical characteristics and fatty acid profile of meat from lambs with different genotypes and diets. R Bras Zootec. 2015; 44(7):248-254. http://dx.doi.org/10.1590/ $\underline{\text { S1806-92902015000700003 }}$

25. Souza LL, Azevêdo JAG, Araújo GGL, SantosCruz CL, Cabral ÍS, Almeida FM, et al. Crude glycerin for Santa Inês and F1 Dorper $\times$ Santa Inês lambs. Small Rumin Res. 2015; 129:1-5. https://doi.org/10.1016/j. smallrumres.2015.06.006
26. Cartaxo FQ, Sousa WH, Costa RG, Cezar MF, Pereira Filho JM, Cunha MGG. Características quantitativas da carcaça de cordeiros de diferentes genótipos submetidos a duas dietas. R Bras Zootec. 2011; 40(10):22202227. http://dx.doi.org/10.1590/S1516$\underline{35982011001000023}$

27. Costa RG, Araújo Filho JT, Sousa WH, Gonzaga Neto S, Madruga MS, Fraga AB. Effect of diet and genotype on carcass characteristics of feedlot hair sheep. R Bras Zootec. 2010; 39(12):2763-2768. http://dx.doi. org/10.1590/S1516-35982010001200029

28. Batista ASM, Costa RG, Garruti DS, Madruga MS, Queiroga RCRE, Araújo Filho JT. Effect of energy concentration in the diets on sensorial and chemical parameters of Morada Nova, Santa Inez and Santa Inez $\times$ Dorper lamb meat. R Bras Zootec. 2010; 39(9):2017-2023. http://dx.doi. org/10.1590/S1516-35982010000900021

29. Costa RG, Batista ASM, Azevedo PS, Queiroga RCRE, Madruga MS, Araújo Filho JT. Lipid profile of lamb meat from different genotypes submitted to diets with different energy levels. R Bras Zootec. 2009; 38(3):532-538. https://doi.org/10.1590/ $\underline{\mathrm{S} 1516-35982009000300019}$

30. Madruga MS, Araújo WO, Sousa WH, Cezar MF, Galvão MS, Cunha MGG. Efeito do genótipo e do sexo sobre a composição química e o perfil de ácidos graxos da carne de cordeiros. R Bras Zootec. 2006; 35(4):1838-1844. http://dx.doi. org/10.1590/S1516-35982006000600035

31. Moreno GMB, Buzzulini C, Borba $H$, Costa AJ, Lima TMA, Dourado JFB. Efeito do genótipo e do teor de proteína da dieta sobre a qualidade da carne de cordeiros. Rev Bras Saúde Prod Anim. 2011; 12(3):630-640. http://www.rbspa.ufba.br/index.php/rbspa/ article/viewArticle/2085

32. Senegalhe FBD, Macedo FAF, Mora NHAP, Gualda TP, Radis AC, Queiroz EO, et al. Composição química da carne de cordeiros abatidos com diferentes espessuras de gordura subcutânea. Rev Bras Saúde Prod Anim. 2014; 15(3): 740-753. http://dx.doi. org/10.1590/S1519-99402014000300022 
Vega-Britez et al - Canal y carne de corderos Santa Inés y cruces con Dorper o Texel

33. Cartaxo FQ, Sousa WH, Cezar MF, Gonzaga Neto S, Cunha MGG. Efeitos do genótipo e da condição corporal sobre o desempenho de cordeiros terminados em confinamento. R Bras Zootec. 2008; 37(8):14831489. https://doi.org/10.1590/S1516$\underline{35982008000800021}$

34. Esteves GIF, Peripolli V, Costa Jr BG, Tanure $C B$, Menezes AM, Souza JR, et al. Effects of genetic group, pregnancy and age on carcass traits, meat quality and fatty acid profile in female sheep. Rev Colomb Cienc Pecu. 2019; 32(1):21-33. http://dx.doi. org/10.17533/udea.rccp.v32n1a03

35. Freire MTA, Nakao MY, Guerra CC, Souza SC, Trindade MA. Determinação de parâmetros físico-químicos e de aceitação sensorial da carne de cordeiros proveniente de diferentes tipos raciais. Alim Nutr. 2010; 21(3):481486. http://serv-bib.fcfar.unesp.br/seer/ index.php/alimentos/article/viewArticle/909

36. Garcia IFF, Perez JRO, Oliveira MV. Características de carcaça de cordeiros Texel x Bergamácia, Texel x Santa Inês e Santa Inês puros, terminados em confinamento, com casca de café como parte da dieta. $\mathrm{R}$ Bras Zootec. 2000; 29(1):253-260. https://doi. org/10.1590/S1516-35982000000100033

37. Garcia IFF, Perez JRO, Teixeira JC, Barbosa CMP. Desempenho de cordeiros Texel $x$ Bergamácia, Texel x Santa Inês e Santa Inês puros, terminados em confinamento, alimentados com casca de café como parte da dieta. R Bras Zootec. 2000; 29(2):564572. https://doi.org/10.1590/S1516$\underline{35982000000200033}$

38. Garcia IFF, Perez JRO, Teixeira JC. Componentes de carcaça e composição de alguns cortes de cordeiros Texel $x$ Bergamácia, Texel x Santa Inês e Santa Inês puros, terminados em confinamento, com casca de café como parte da dieta. R Bras Zootec. 2003; 32(6):1999-2006. https://doi. org/10.1590/S1516-35982003000800025

39. Garcia IFF, Perez JRO, Bonagurio S, Assis RM, Carneiro e Pedreira B, Souza XR. Desempenho de Cordeiros Santa Inês Puros e Cruzas Santa Inês com Texel, Ile de France e Bergamácia. R Bras Zootec. 2004; 33(6):1591-1603. https://doi.org/10.1590/ $\underline{\mathrm{S} 1516-35982004000600027}$
40. Issakowicz J, Bueno MS, Issakowicz ACKS, Haguiwara MMH. Características quantitativas da carcaça e qualitativas da carne de cordeiros Morada Nova, Santa Inês e $1 / 2$ Ile de France $1 / 2$ Texel terminados em confinamento. B Indústr Anim. 2014; 71(3):217-225. https://doi.org/10.17523/ bia.v71n $3 p 217$

41. Landim AV, Mariante AS, McManus C, Gugel $\mathrm{R}$, Paiva SR. Características quantitativas da carcaça, medidas morfométricas e suas correlações em diferentes genótipos de ovinos. Ciênc Anim Bras. 2007; 8(4):665676. https://www.alice.cnptia.embrapa.br/ bitstream/doc/189328/1/ID292091.pdf

42. Maia MO, Costa FS, Susin I, Rodrigues GH, Ferreira EM, Pires AV, et al. Efeito do genótipo sobre a composição química e o perfil de ácidos graxos da carne de borregas. R Bras Zootec. 2012; 41(4):986-992. https://doi. org/10.1590/S1516-35982012000400022

43. Quirino $C R$, Azevedo AS, Madella-Oliveira AF, Pacheco A, Costa RLD, Beltrame RT. Características de carcaça em cordeiros Santa Inês e cruzados $1 / 4$ Santa Inês e 3/4 Dorper de Brasil. AICA. 2015; 6(1):552-561. https:// aicarevista.jimdo.com/n\%C3\%BAmeros/ descargas-pdf/colombia-2015/

44. Santos RS, Ribeiro KG, Valadares Filho SC, Pereira OG, Villela SDJ, Rennó LN, et al. Effects of diets with high and low protein contents and two concentrate levels in Santa Ines x Texel lambs. Livest Sci. 2015; 177:79-87. https://doi.org/10.1016/j. livsci.2015.04.011

45. Souza DA, Selaive-Villarroel AB, Pereira ES, Osório JCS, Teixeira A. Growth performance, feed efficiency and carcass characteristics of lambs produced from Dorper sheep crossed with Santa Inês or Brazilian Somali sheep. Small Rumin Res. 2013; 114:51-55. http://dx.doi.org/10.1016/j. smallrumres.2013.06.006

46. Cartaxo FQ, Sousa WH. Correlações entre as características obtidas in vivo por ultrasom e as obtidas na carcaça de cordeiros terminados em confinamento. R Bras Zootec. 2008; 37(8):1490-1495. https://doi. org/10.1590/S1516-35982008000800022 
47. Osório JCS, Osório MTM, Vargas Junior FM, Fernandes ARM, Seno LO, Ricardo HA, et al. Critérios para abate do animal e a qualidade da carne. Rev Agrarian. 2012; 5(18):433443. http://ojs.ufgd.edu.br/index.php/ agrarian/article/view/1822

48. Costa RS, Henriques LSV, Tavares EM, Costa Henry F, Quirino CR. Qualidade da carne de cordeiros Santa Inês e F1 Santa Inês X Dorper. Rev Bras Med Vet. 2014; 36(3):337343. http://rbmv.org/index.php/BJVM/ article/view/531
49. Landim AV, Costa HHA, Carvalho FC, Costa AC, Alencar RT, Silva LNC, et al. Desempenho produtivo e características de carcaça de cordeiros Rabo Largo puro e cruzados com Santa Inês. Arq Bras Med Vet Zootec. 2017; 69(5):1267-1274. http:// dx.doi.org/10.1590/1678-4162-9210 